\title{
Estrutura populacional, tamanho e razão sexual de Podocnemis unifilis (Testudines, Podocnemididae) no rio Guaporé (RO), norte do Brasil
}

\author{
Augusto Fachín-Terán ${ }^{1,2,3,4}$ and Richard C. Vogt ${ }^{4}$ \\ 1 Escola Normal Superior, Universidade do Estado do Amazonas, Av. Darcy Vargas 2490, 69050-020, Manaus, AM, Brasil. \\ E-mail: fachinteran@yahoo.com.br. \\ 2 Departamento de Ciências Fundamentais e Desenvolvimento Agrícola, Faculdade de Ciências Agrárias, Universidade \\ Federal do Amazonas, Av. General Rodrigo Octávio Jordão Ramos, 3000, 69077-000, Manaus, AM, Brasil. \\ 3 Departamento Académico de Ecología y Manejo, Facultad de Ciencias Biológicas, Universidad Nacional de la Amazonía \\ Peruana. Casilla Postal 496, Iquitos, Loreto-Perú. \\ ${ }^{4}$ Instituto Nacional de Pesquisas da Amazônia - INPA. Coordenação de Pesquisa em Biologia de Água Doce. Alameda \\ Cosme Ferreira, 1756, 69011-970, Manaus, AM, Brasil. E-mail: vogt@inpa.gov.br.
}

\begin{abstract}
Population structure, size and sex ratio in Podocnemis unifilis (Testudines, Podocnemididae) in the Guaporé river (RO), northern Brazil. Population structure and sex ratio of Podocnemis unifilis was studied from June to November 1989 and from March to June 1990 in the Guaporé river and its tributaries São Domingos and São Miguel, in the municipality of Costa Marques, Rondônia, Brazil. The margins of these rivers are not altered much by human activities and the water is not polluted in any way. Turtles were captured using 12 fyke nets. Around $1 \%$ of the captured turtles died using this method. The majority of the turtles was captured in the bays and oxbows $(55.8 \%)$ and when the water level of the river was declining $(\mathrm{N}=785)$. The proportion of adults $(73.4 \%)$ in the sample was about three times higher than that of juveniles (26.6\%). The distribution of carapace length in males formed a normal curve, while that of females showed no well-defined pattern. The sex ratio of adults in this population was 9.8 males per female.
\end{abstract}

Keywords: Testudines, Podocnemididae, Podocnemis unifilis, biometry, population structure, sex ratio, Rondônia, Brazil.

Palavras-chave: Testudines, Podocnemididae, Podocnemis unifilis, biometria, estrutura populacional, razão sexual, Rondônia, Brasil.

\section{Introdução}

O programa de preservação de quelônios na Amazônia Legal Brasileira completou 28 anos em 2003. Nesse período, suas atividades estive-

Recebido em 12 de janeiro de 2004.

Aceito em 19 julho de 2004.

Distribuído em 30 de setembro de 2004. ram direcionadas prioritariamente à preservação de Podocnemis expansa e, em menor escala, de $P$. unifilis, $P$. sextuberculata, $P$. erythrocephala e Peltocephalus dumerilianus, sendo liberados nos rios da Amazônia milhares de filhotes dessas espécies (Cantarelli 1997). Esse esforço do Governo Federal foi importante para recuperar e manter as populações de quelônios nas áreas naturais. Para atingir esses objetivos, é 
realizado o monitoramento permanente dos locais de reprodução, do número de covas e da produção de filhotes, aumentando-se, dessa forma, o sucesso reprodutivo das espécies. Os estudos sobre ecologia reprodutiva demonstraram que os filhotes de Podocnemis spp. têm determinação do sexo dependente da temperatura de incubação dos ovos (Alho et al. 1984, 1985, Souza e Vogt 1994, Valenzuela et al. 1997, Von Hildebrand et al. 1997, Paez e Bock 1998, Pezzuti e Vogt 1999, Valenzuela 2001). Em função do desconhecimento do status atual de conservação das populações dessas espécies nas áreas de influência do Projeto Quelônios da Amazônia/RAN/IBAMA, faz-se necessária a coleta de informações referentes à biologia e à ecologia de suas populações.

Apesar do gênero Podocnemis ser um dos mais estudados, a maioria dos trabalhos referese à ecologia reprodutiva, sendo $P$. unifilis uma das espécies mais estudadas (Fachín-Terán e Von Mülhen 2003). Nos últimos cinco anos, foram geradas inúmeras informações sobre parâmetros populacionais de Podocnemis spp. por meio de dados de caça (Fachín-Terán et al. 2000, Soares 2000) e da amostragem direta das populações (Bataus 1998, Fachín-Terán et al. 2003) da Amazônia brasileira.

Após mais de 36 anos de promulgada a Lei $\mathrm{n}^{\circ} 5.197 / 67$, que dispõe sobre a Proteção da Fauna, proibindo a livre captura de animais silvestres, as comunidades ribeirinhas mantêm o hábito cultural de alimentar-se e de comercializar quelônios (Rebelo e Lugli 1996, FachínTerán et al. 2000, Soares 2000, Fachín-Terán 2003), ocasionando, muitas vezes, repreensões pelos órgãos de fiscalização do governo. As instituições de fiscalização e de pesquisa devem dar respostas para que as populações dessas espécies sejam manejadas sustentavelmente pelas populações ribeirinhas que participam do programa de preservação.

A carência de dados populacionais sobre $P$. unifilis dificulta a determinação de sua situação atual e a implantação de práticas de manejo e conservação dessa espécie no rio Guaporé e tributários. O presente trabalho teve como objetivo determinar a estrutura populacional, o tamanho e a razão sexual de Podocnemis unifilis (Troschel, 1848) no rio Guaporé, Rondônia, norte do Brasil.

\section{Material e Métodos}

\section{Área de estudo}

O rio Guaporé e seus tributários localizamse no município de Costa Marques, Rondônia, Brasil (12³0'S, 64'10'W), tendo sua nascente na Serra dos Parecis, estado de Mato Grosso (Figuras 1 e 2). Suas águas são de cor negra, sem poluição, com margens pouco perturbadas. Tem um curso de aproximadamente $1210 \mathrm{~km} \mathrm{e}$ faz a divisa do Brasil com a Bolívia no estado de Rondônia. Em ambas as margens desse rio, encontram-se muitos lagos, conhecidos localmente como "baías", que se conectam com o rio durante a cheia. Durante a época da vazante (setembro a novembro), algumas baías ficam isoladas e outras mantêm a comunicação com o rio Guaporé. A partir do mês de junho, afloram praias (tabuleiros) de areia por todo o seu curso, onde as fêmeas de $P$. expansa e $P$. unifilis nidificam. $\mathrm{O}$ rio Guaporé e seus tributários estão sujeitos a inundações periódicas, de intensidade que varia dependendo dos ciclos pluviométricos anuais.

O rio São Miguel é afluente da margem direita do rio Guaporé. Nasce na Serra dos Parecis e têm um curso de aproximadamente 195 km em direção geral NE-SW, com uma largura máxima de $100 \mathrm{~m}$. Em ambas as margens, encontra-se uma vegetação aberta denominada de campos naturais. Os rios Guaporé e São Miguel apresentam muitos lagos em ambas as margens, com abundância de macrófitas, incluindo Eichhornia (Pontederiaceae), Paspalum (Poaceae) e ciperáceas. O rio São Domingos é afluente da margem direita do rio Guaporé, nasce na Serra de Uopiane e tem um curso de aproximadamente $165 \mathrm{~km}$ em direção geral NE-SW (Roque 1968). Não possui lagos, e suas águas são de cor negra. Na época da seca, 
sua largura é menor que $20 \mathrm{~m}$. Possui abundante vegetação aquática, principalmente Eichhornia, que cobre completamente grandes segmentos do rio. O rio Guaporé e tributários abrigam uma diversidade de hábitats dos quais alguns só aparecem sazonalmente. Algumas denominações desses hábitats só são usadas localmente: canos, canais que drenam as baías; baías, lagos da planície inundada, paraná, ramo do canal principal de um rio que forma um braço que atravessa a floresta inundada, conectando-se a jusante ao mesmo corpo de água.

\section{Metodologia}

As capturas de Podocnemis unifilis foram realizadas de junho a novembro de 1989 e de março a junho de 1990, com o auxílio de 12 armadilhas tipo fyke net (Figura 3). Essas armadilhas são feitas com argolas de ferro com diâmetro de 0,9 a $2 \mathrm{~m}$ e comprimento de 3 a 4 $\mathrm{m}$, com entrada do tipo funil. Essa estrutura é unida a malhas de 9 a $14 \mathrm{~m}$ de comprimento com 1 a $2 \mathrm{~m}$ de largura (5 cm entre os nós) e que apresentam flutuadores na parte superior e chumbo na parte inferior. As armadilhas foram geralmente instaladas em locais rasos, onde o chumbo do painel de malha pudesse tocar o fundo para tornar a armadilha mais eficiente. Os quelônios foram capturados devido ao seu movimento natural, já que a malha atua como uma barreira que conduz os animais para dentro do funil (Vogt 1980). Em nenhuma oportunidade foram usadas iscas para capturar os animais.

Com a finalidade de capturar animais de ambos os sexos e de vários tamanhos, as 12 armadilhas foram deixadas sistematicamente em cada um dos seguintes hábitats: floresta inundada, riachos de floresta, beira de rios e lagos. O comprimento e a altura das armadilhas restringiram as capturas a águas rasas perto das margens. O diâmetro das malhas permitiu somente a captura de indivíduos com largura da carapaça superior a $5 \mathrm{~cm}$. As armadilhas foram visitadas periodicamente pela manhã e à tarde em intervalos de 12 horas, sendo transferidas para outros locais após uma amostragem de 48 horas. Os animais capturados foram transportados ao acampamento temporário, situado a pequena distância do local de amostragem, onde foram pesados, medidos, sexados e marcados. As medidas da carapaça foram realizadas em linha reta, no ponto de maior amplitude (Medem 1976). O sexo foi identificado pelo tamanho, cor da cabeça, largura da carapaça, forma do plastrão, fenda da placa anal, distância pré-cloacal e comprimento e espessura da cauda (Ponce 1979, Pritchard e Trebbau 1984). Cada animal foi marcado permanentemente com um corte de forma retangular no centro do escudo marginal da carapaça com um código previamente estabelecido (Cagle 1939). Logo após a tomada dos dados e marcações, os animais foram liberados no local de captura.

Segundo Gibbons (1990), a razão sexual deve ser calculada com base nos indivíduos que alcançaram a maturidade sexual. Com base nas observações de Soares (2000) para essa espécie no rio Guaporé, foram considerados adultos os indivíduos com comprimento linear da carapaça $\geq 25 \mathrm{~cm}$, para os machos, ou $\geq 35 \mathrm{~cm}$, para as fêmeas; foram considerados juvenis os indivíduos com carapaças menores que esses valores para cada sexo.

Como se desconhece a idade em função do tamanho dessa espécie em áreas naturais, estabelecemos arbitrariamente classes de tamanho com intervalos de $2 \mathrm{~cm}$ de comprimento da carapaça, que foram usadas nas análises da estrutura populacional. Como não existem informações sobre a estrutura populacional e com a finalidade de comparar a freqüência de tamanhos de $P$. unifilis, construímos um gráfico com dados de caça de Fachín-Terán et al. (2000) coletados no setor Jarauá da Reserva de Desenvolvimento Sustentável Mamirauá.

O teste de Qui-quadrado foi usado para comparar o número de animais de ambos os sexos capturados na floresta inundada e o número de animais capturados nos diferentes hábitats durante a enchente e a vazante. Também 


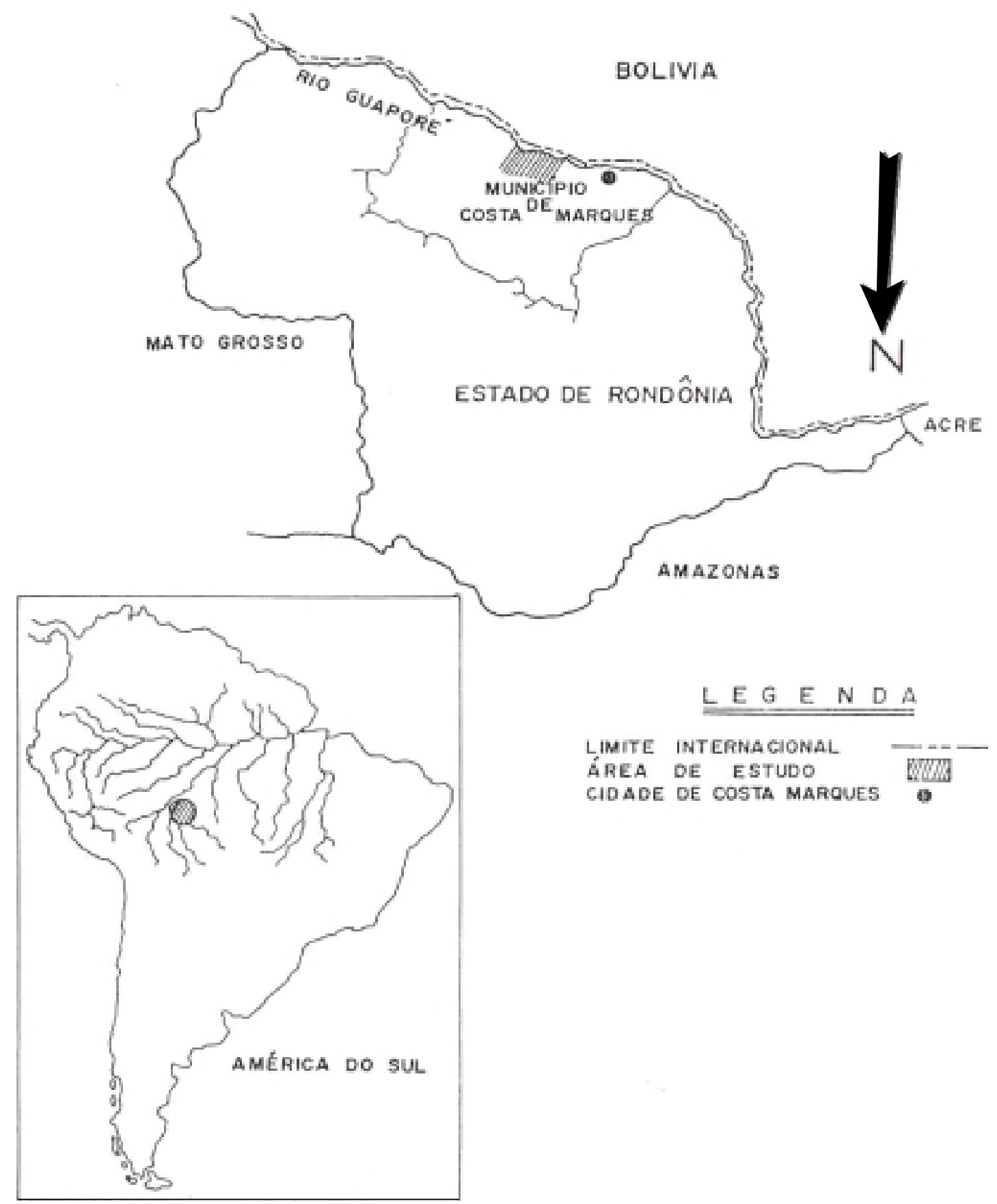

Figura 1 - Localização da área de estudo no rio Guaporé, Costa Marques, Rondônia, Brasil. 


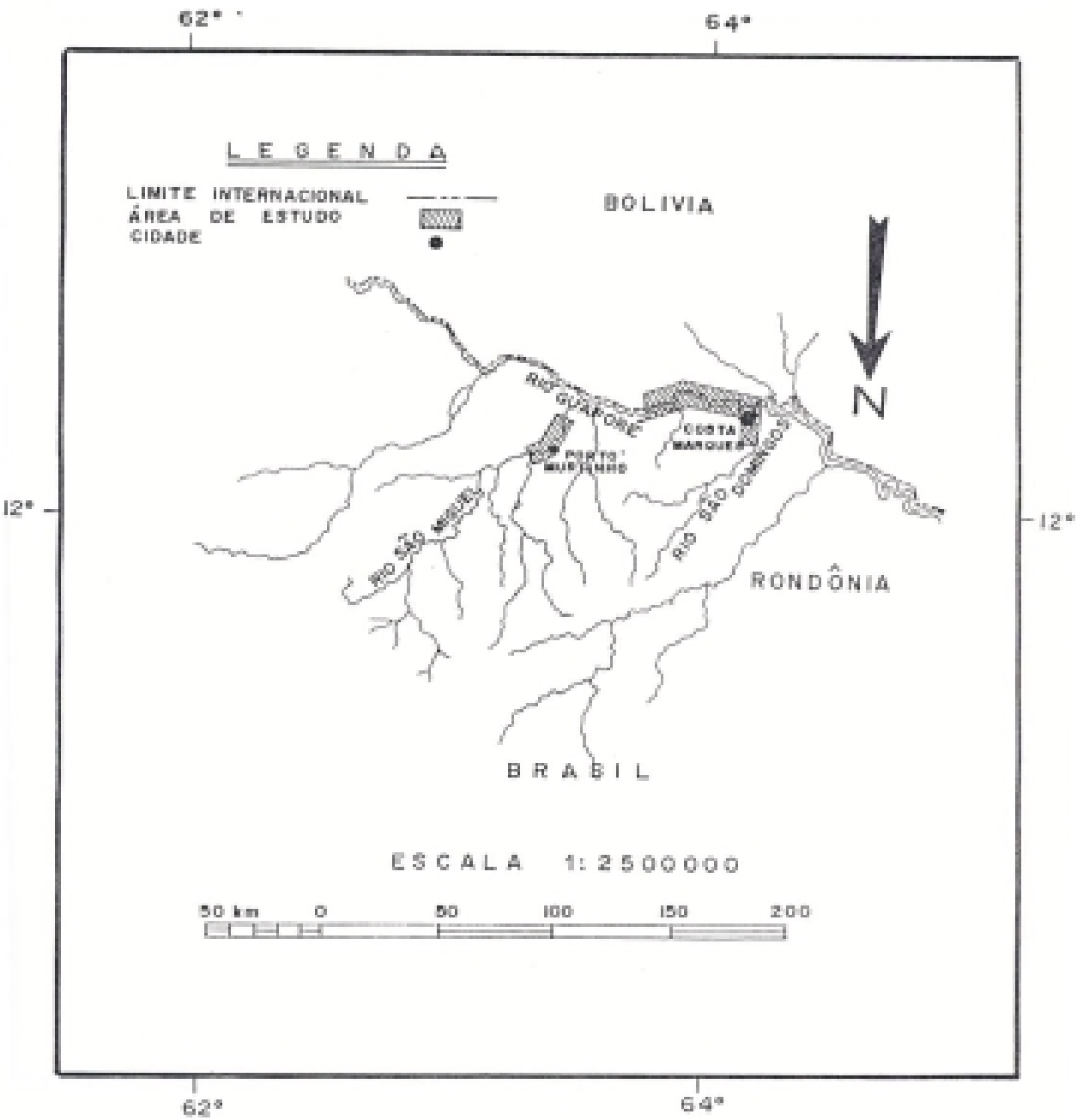

Figura 2 - Localização da área de estudo nos rios São Domingos, Guaporé e São Miguel.

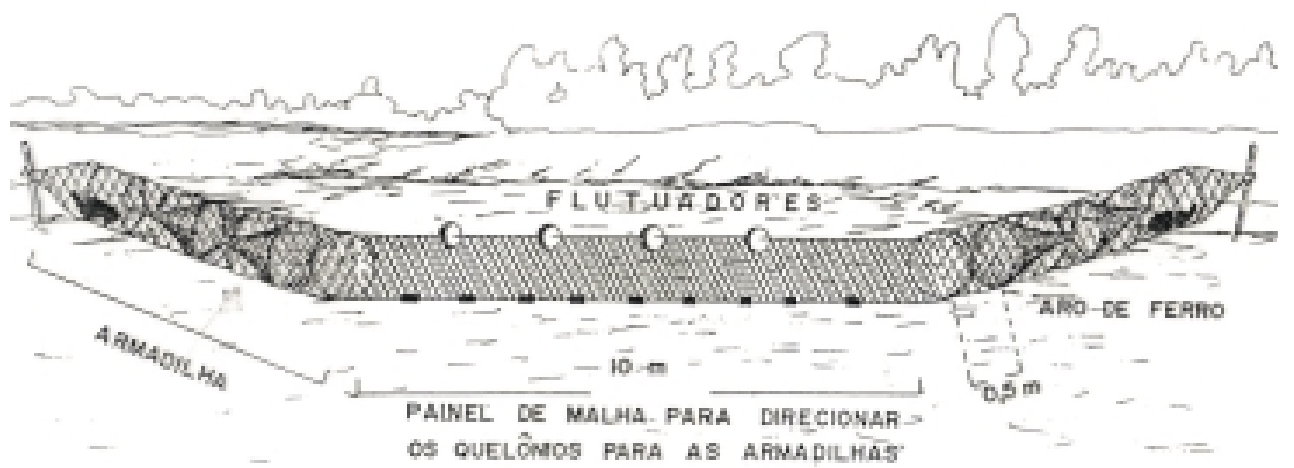

Figura 3 - Representação esquemática de uma armadilha do tipo fyke net. 
usamos esse teste para comparar a razão sexual para os indivíduos capturados nos locais amostrados. As análises estatísticas foram feitas no programa STATISTIX for Windows (Analytical Software 2000).

\section{Resultados}

Durante os 10 meses de amostragem, capturamos 837 animais, sendo 747 machos, 88 fêmeas e 2 cujo sexo não foi determinado em função do tamanho e características sexuais secundárias externas duvidosas. Desses, somente 46 machos (5\%) foram recapturados uma vez. Fêmeas não foram recapturadas. As capturas ocorreram principalmente quando o nível da água estava diminuindo (Figura 4).

A maioria dos animas foi capturada nos rios São Miguel (N=333) e Guaporé (N=499) (Tabela 1$)$. As capturas ocorreram principalmente durante a vazante $(\mathrm{N}=785)$. No rio São Domingos, foram capturados apenas cinco indivíduos devido à dificuldade de acesso durante a época seca.

Podocnemis unifilis apresenta dimorfismo sexual no tamanho e nas seguintes características externas: fenda da placa anal, distância pré-cloacal e comprimento e espessura da cauda. Os machos foram menores do que as fêmeas (Tabela 2). O comprimento médio da carapaça de 747 machos foi de 26,4 $\pm 3,28 \mathrm{~cm}$ (amplitude: $9,8-39,6 \mathrm{~cm}$ ). O peso médio foi de $2,254 \pm 0,69$ $\mathrm{kg}(0,15-4,3 \mathrm{~kg})$. O comprimento médio da carapaça de 88 fêmeas foi de $35,0 \pm 7,73 \mathrm{~cm}$ $(12,4-46,5 \mathrm{~cm})$. O peso médio foi de $5,769 \pm 2,74 \mathrm{~kg}(0,3-11,2 \mathrm{~kg})$. O tamanho relatado foi baseado em todos os indivíduos capturados.

Os animais foram encontrados em diferentes tipos de hábitats, tendo sido capturados principalmente nas baías $(55,8 \%)$ e, em menor número, no rio $(17,7 \%)$ e na floresta inundada $(26,5 \%)$ (Tabela 3$)$. A captura dessa espécie ocorreu principalmente em águas rasas, de 2 a $3 \mathrm{~m}$ de profundidade. O número de animais capturados nos diferentes hábitats durante a

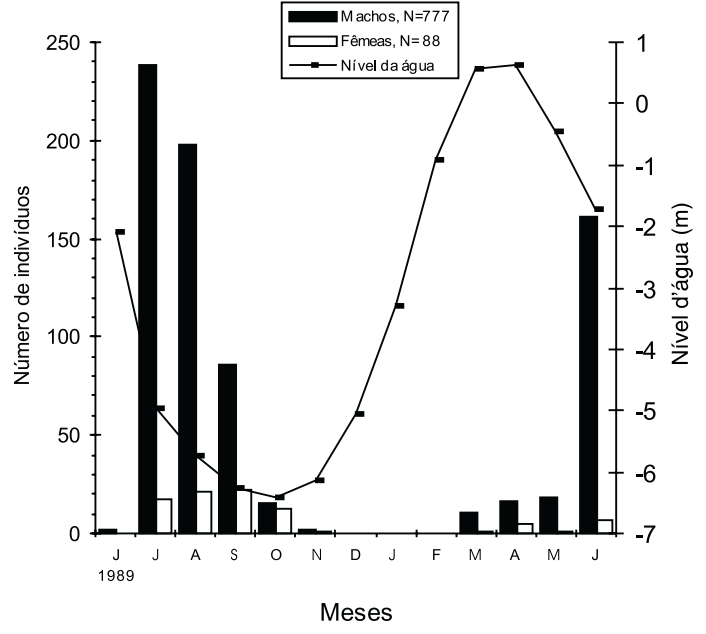

Figura 4 - Número de indivíduos capturados (barras) durante os meses da amostragem no rio Guaporé e tributários e variação do nível d'água.

enchente e a vazante foi significativamente diferente $\left(\chi^{2}=154,07 ; \mathrm{df}=2 ; \mathrm{p}=0,00\right)$. O número de animais de ambos os sexos capturados na floresta inundada durante a enchente e a vazante foi significativamente diferente $\left(\chi^{2}=7,06 ; \mathrm{df}=1\right.$; $\mathrm{p}=0,00)$.

Dos 837 exemplares capturados, nove morreram nas armadilhas (1,1\%) (Tabela 4). A morte dos animais ocorreu principalmente por afogamento, isto é, quando o indivíduo ficava preso entre o segundo ou o terceiro compartimento da armadilha e não conseguia atingir o compartimento que permanecia fora da água e que servia de respiradouro. Em uma ocasião, encontramos um macho afogado no compartimento que servia de respiradouro, provavelmente devido à grande quantidade de animais capturados em um dos lados da armadilha (46 machos e uma fêmea), o que teria dificultado seu acesso ao respiradouro.

A maior parte dos animais capturados $(73,4 \%)$ foram adultos de ambos os sexos. Mais de um quarto da amostra foi composta por juvenis $(26,6 \%)$ (Tabela 5). Em relação às faixas etárias por sexo, observou-se uma maior porcen- 
Tabela 1 - Número de indivíduos de Podocnemis unifilis coletados de junho a novembro de 1989 e de março a junho de 1990.

\begin{tabular}{lcccccccc}
\hline \multirow{2}{*}{ Local } & \multicolumn{2}{c}{ Enchente } & Total & \multicolumn{2}{c}{ Vazante } & Total & Vazante & TOTAL \\
\cline { 2 - 9 } & $\sigma^{x}$ & + & & $\sigma^{x}$ & + & & Indeterminado & \\
\hline Rio São Miguel & 1 & & 1 & 312 & 20 & 332 & & 333 \\
Rio Guaporé & 44 & 7 & 51 & 387 & 59 & 446 & 2 & 499 \\
Rio São Domingos & & & & 3 & 2 & 5 & & 5 \\
\hline TOTAL & 45 & 7 & 52 & 702 & 81 & 783 & 2 & 837 \\
\hline
\end{tabular}

Tabela 2 - Morfometria de machos $(\mathrm{N}=747)$ e fêmeas $(\mathrm{N}=88)$ de P. unifilis capturados em rios da região amazônica entre junho e novembro de 1989 e entre março e junho de 1990. CC, comprimento da carapaça; LC, largura da carapaça; ALT, altura do casco; CP, comprimento do plastrão; LCA, largura da cabeça; CPF, comprimento da placa femoral; $\overline{\mathrm{x}}$, média; DP, desvio padrão.

\begin{tabular}{lcccccc}
\hline & \multicolumn{3}{c}{$\sigma^{x}$} & \multicolumn{3}{c}{+} \\
\cline { 2 - 7 } Característica & $\overline{\mathbf{x}}$ & DP & Amplitude & $\overline{\mathbf{x}}$ & $\mathbf{D P}$ & Amplitude \\
\hline CC $(\mathrm{cm})$ & 26,40 & 3,28 & $9,8-39,6$ & 35,03 & 7,73 & $12,4-46,5$ \\
LC (cm) & 20,27 & 2,44 & $8,4-29,0$ & 25,90 & 5,30 & $10,2-33,2$ \\
ALT $(\mathrm{cm})$ & 9,14 & 0,99 & $4,3-11,7$ & 12,46 & 3,24 & $5,0-26,0$ \\
CP $(\mathrm{cm})$ & 23,12 & 2,81 & $5,2-33,2$ & 32,78 & 7,72 & $4,3-42,8$ \\
LCA (cm) & 3,76 & 0,39 & $1,95-5,5$ & 4,76 & 0,82 & $2,44-6,23$ \\
CPF (cm) & 4,82 & 0,63 & $1,9-6,6$ & 6,62 & 1,53 & $2,4-8,9$ \\
Peso (kg) & 2,25 & 0,69 & $0.15-4,3$ & 5,76 & 2,74 & $0,3-11,2$ \\
\hline
\end{tabular}

Tabela 3 - Número de indivíduos de P. unifilis capturados durante a enchente e a vazante nos diferentes hábitats amostrados no rio Guaporé e tributários entre junho e novembro de 1989 e entre março e junho de 1990.

\begin{tabular}{|c|c|c|c|c|c|c|c|c|c|c|}
\hline \multirow[b]{2}{*}{ HÁBITATS } & \multicolumn{4}{|c|}{ Enchente } & \multicolumn{4}{|c|}{ Vazante } & \multicolumn{2}{|c|}{ TOTAL } \\
\hline & $\sigma^{x}$ & 우 & $\mathrm{N}$ & $\%$ & $\sigma^{x}$ & 우 & $\mathrm{N}$ & $\%$ & $\mathrm{~N}$ & $\%$ \\
\hline Rio & & & & & 119 & 29 & 148 & 18,9 & 148 & 17,7 \\
\hline Floresta inundada & 45 & 7 & 52 & 100 & 163 & 6 & 169 & 21,6 & 221 & 26,5 \\
\hline Baía & & & & & 420 & 46 & 466 & 59,5 & 466 & 55,8 \\
\hline TOTAL & 45 & 7 & 52 & 100 & 702 & 81 & 783 & 100 & 835 & 100 \\
\hline
\end{tabular}

tagem de machos $(74,4 \%)$ e fêmeas $(64,8 \%)$ adultas. O número de animais adultos de ambos os sexos foi maior no rio São Miguel em relação ao rio Guaporé. A porcentagem de machos juvenis no rio Guaporé foi o dobro $(32,5 \%)$ daquela capturada no rio São Miguel (16\%); para as fêmeas juvenis, a porcentagem foi um pouco maior no rio Guaporé $(36,9 \%)$ do que no rio São Miguel (33,3\%) (Tabela 6).

O tamanho dos indivíduos (comprimento da carapaça) de Podocnemis unifilis da população do rio Guaporé e tributários apresentou distri- 
Tabela 4 - Porcentagem de indivíduos de Podocnemis unifilis mortos por diferentes causas durante o período de estudo.

\begin{tabular}{|c|c|c|c|c|c|c|c|c|}
\hline \multirow[t]{2}{*}{ Causa da morte } & \multicolumn{2}{|c|}{$0^{x}$} & \multicolumn{2}{|c|}{ ㅇ } & \multicolumn{2}{|c|}{ Indeterminado } & \multicolumn{2}{|c|}{ TOTAL } \\
\hline & $\mathrm{N}$ & $\%$ & $\mathrm{~N}$ & $\%$ & $\mathrm{~N}$ & $\%$ & $\mathrm{~N}$ & $\%$ \\
\hline Vivos & 734 & 98,3 & 83 & 94,3 & 2 & 100 & 819 & 97,8 \\
\hline Armadilha & 7 & 0,9 & 2 & 2,3 & & & 9 & 1,1 \\
\hline No saco & 1 & 0,1 & & & & & 1 & 0,1 \\
\hline Pesquisa & 5 & 0,7 & 3 & 3,4 & & & 8 & 1,0 \\
\hline TOTAL & 747 & 100 & 88 & 100 & 2 & 100 & 837 & 100 \\
\hline
\end{tabular}

Tabela 5 - Porcentagem de juvenis e adultos de Podocnemis unifilis capturados em rios da região amazônica entre junho e novembro de 1989 e entre março e junho de 1990 segundo o sexo e as faixas etárias.

\begin{tabular}{lccccccc}
\hline & \multicolumn{3}{c}{$\sigma^{\boldsymbol{*}}$} & \multicolumn{2}{c}{$\%$} & \multicolumn{2}{c}{ TOTAL } \\
\cline { 2 - 7 } IDADE & $\mathbf{N}$ & $\boldsymbol{\%}$ & $\mathbf{N}$ & $\boldsymbol{\%}$ & $\mathbf{N}$ & $\boldsymbol{\%}$ \\
\hline Juvenis & 191 & 25,6 & 31 & 35,2 & 222 & 26,6 \\
Adultos & 556 & 74,4 & 57 & 64,8 & 613 & 73,4 \\
\hline
\end{tabular}

Tabela 6 - Porcentagem de juvenis e adultos de Podocnemis unifilis capturados nos rios Guaporé e São Miguel entre junho e novembro de 1989 e entre março e junho de 1990 segundo o sexo e as faixas etárias.

\begin{tabular}{|c|c|c|c|c|c|c|c|c|c|c|c|c|}
\hline \multirow[b]{2}{*}{ IDADE } & \multicolumn{6}{|c|}{ Rio Guaporé } & \multicolumn{6}{|c|}{ Rio São Miguel } \\
\hline & $\sigma^{x}$ & $\%$ & 우 & $\%$ & $\mathbf{N}$ & $\%$ & $\sigma^{x}$ & $\%$ & 우 & $\%$ & $\mathbf{N}$ & $\%$ \\
\hline Juvenis & 140 & 32,5 & 24 & 36,9 & 164 & 33,1 & 50 & 16 & 7 & 33,3 & 57 & 17,1 \\
\hline Adultos & 291 & 67,5 & 41 & 63,1 & 332 & 66,9 & 263 & 84 & 14 & 66,7 & 277 & 82,9 \\
\hline TOTAL & 431 & 100 & 65 & 100 & 496 & 100 & 313 & 100 & 21 & 100 & 334 & 100 \\
\hline
\end{tabular}

buição normal para os machos, com tamanhos mais freqüentes nas classes entre 22 e $26 \mathrm{~cm}$ (Figura 5). Apesar de as fêmeas não terem apresentado um padrão definido, as maiores freqüências ocorreram entre as classes de tamanho de 38 e $40 \mathrm{~cm}$. No setor Jarauá da Reserva de Desenvolvimento Sustentável Mamirauá, os tamanhos mais freqüentemente capturados pertenciam às classes de 22 e $26 \mathrm{~cm}$, para os machos, e de 36 a $40 \mathrm{~cm}$, no caso das fêmeas (Figura 6) (Fachín-Terán et al. 2000).
Quando analisamos separadamente as distribuições nos rios Guaporé e São Miguel, o padrão foi quase similar em ambos os rios. No caso dos machos, os tamanhos mais freqüentes ocorreram entre 24 e $28 \mathrm{~cm}$, no rio Guaporé, e entre 26 a $30 \mathrm{~cm}$, no rio São Miguel. Nos dois locais, as fêmeas apresentaram um padrão de distribuição semelhante, sendo mais freqüentes os tamanhos entre 38 e $42 \mathrm{~cm}$ (Figura 7).

A razão sexual para cada local amostrado é apresentada na Tabela 7. A razão sexual baseada 


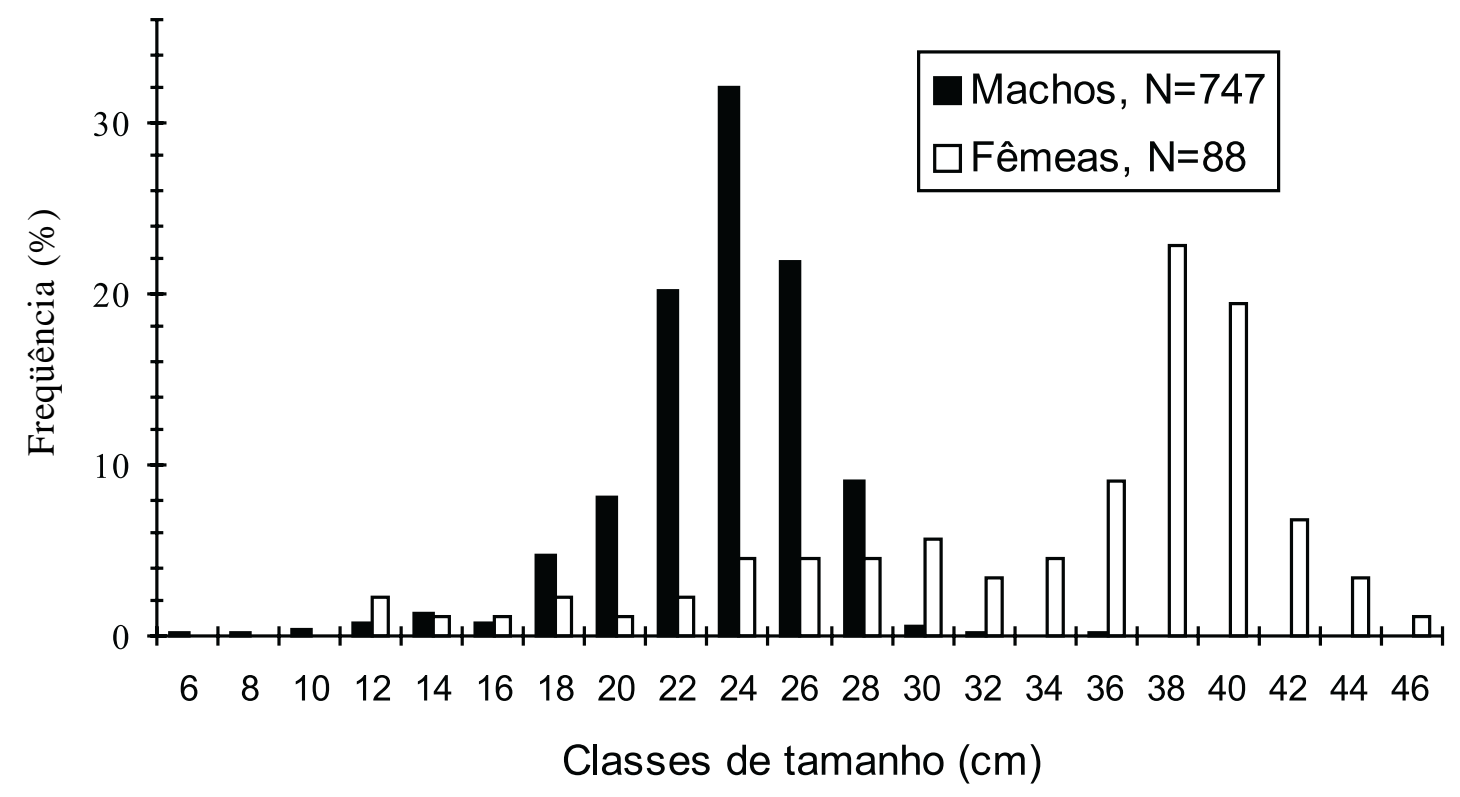

Figura 5 - Distribuição de freqüências de classes de tamanho (comprimento da carapaça) de Podocnemis unifilis no rio Guaporé e tributários (RO).

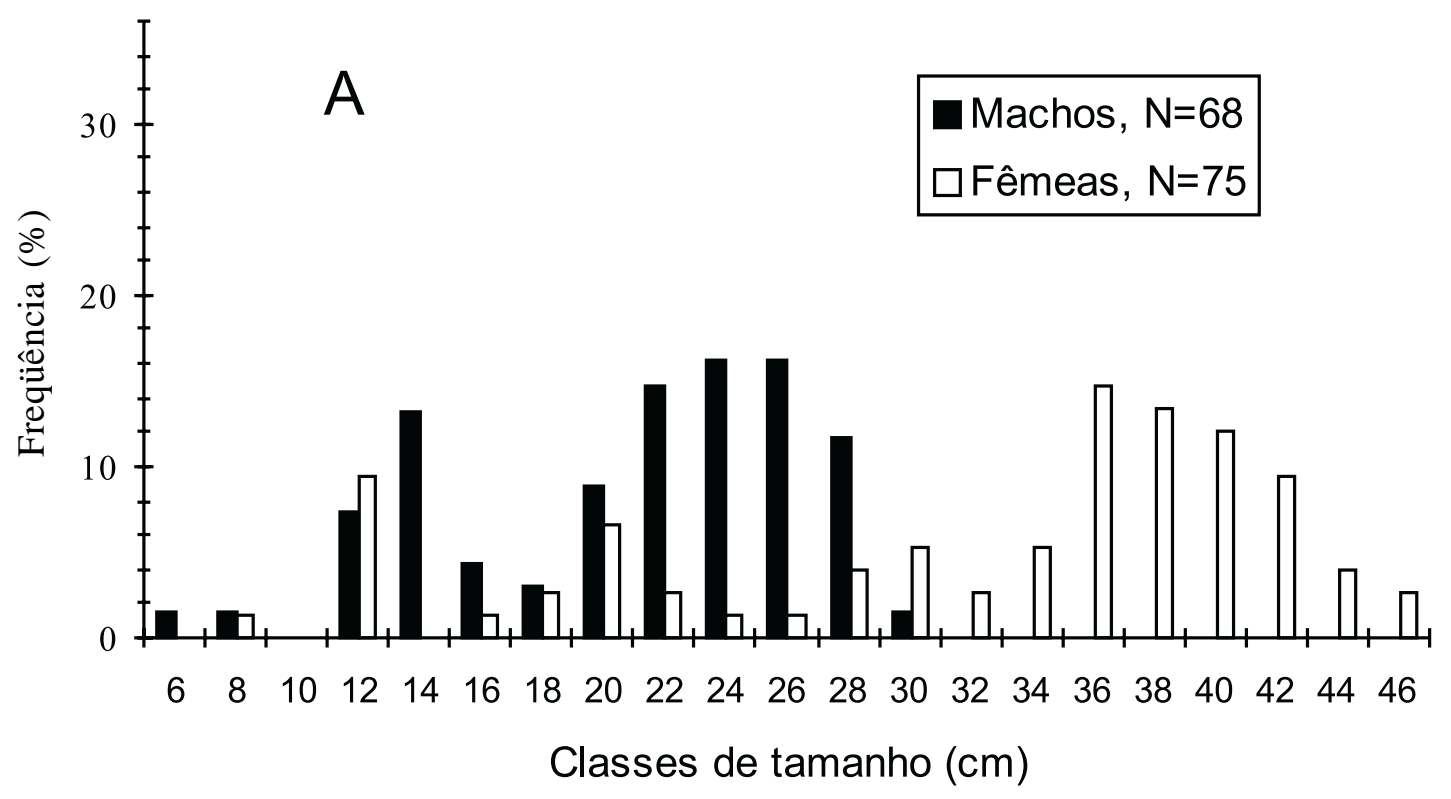

Figura 6 - Distribuição de freqüências de classes de tamanho dos indivíduos de Podocnemis unifilis capturados no setor Jarauá da Reserva de Desenvolvimento Sustentável Mamirauá entre setembro de 1996 e abril de 1998 (segundo dados de Fachín-Terán et al. 2000). 
Tabela 7 - Razão sexual dos indivíduos de Podocnemis unifilis capturados no rio Guaporé e tributários (RO) entre junho e novembro de 1989 e entre março e junho de 1990.

\begin{tabular}{lcccccc}
\hline Local & N & $\begin{array}{c}\text { Razão sexual } \\
\text { baseada em todos } \\
\text { os indivíduos }\end{array}$ & N & $\begin{array}{c}\text { Razão sexual } \\
\text { baseada nos } \\
\text { adultos }\end{array}$ \\
\hline$\sigma^{*}$ & + & & $\sigma^{\star}$ & + & \\
\hline Rio São Miguel & 313 & 20 & $15,7: 1$ & 263 & 14 & $18,8: 1$ \\
Rio Guaporé & 431 & 66 & $6,5: 1$ & 291 & 41 & $7,1: 1$ \\
Rio São Domingos & 3 & 2 & $1,5: 1$ & 2 & 2 & $1: 1$ \\
\hline TOTAL & $\mathbf{7 4 7}$ & $\mathbf{8 8}$ & $\mathbf{8 , 5 : 1}$ & $\mathbf{5 5 6}$ & $\mathbf{5 7}$ & $\mathbf{9 , 8 : 1}$ \\
\hline
\end{tabular}

em todos os indivíduos de Podocnemis unifilis capturados nos três locais amostrados foi de 15,7:1 (rio São Miguel), 6,5:1 (rio Guaporé) e 1,5:1 (rio São Domingos). A razão sexual baseada em adultos que alcançaram a maturidade sexual para toda a área de estudo foi de 9,8:1, desviada para os machos. Tanto a razão sexual baseada em todos os indivíduos capturados como aquela baseada apenas nos adultos foram significativamente diferentes entre os rios São Miguel e Guaporé $\left(\chi^{2}=11,36 ; \mathrm{df}=1\right.$; $\mathrm{p}=0,00$ e $\chi^{2}=9,78 ; \mathrm{df}=1 ; \mathrm{p}=0,00$, respectivamente). A razão sexual baseada em todos os indivíduos capturados e aquela baseada em adultos foram semelhantes para os rios São Miguel $\left(\chi^{2}=0,26 ; \mathrm{df}=1 ; \mathrm{p}=0,60\right)$ e Guaporé $\left(\chi^{2}=0,15 ; \mathrm{df}=1 ; \mathrm{p}=0,69\right)$.

\section{Discussão}

Durante o inicio da época reprodutiva, machos e fêmeas de $P$. expansa e $P$. sextuberculata iniciam a migração saindo dos lagos e da floresta inundada à procura de praias para desovarem coletivamente (Bataus 1998, FachínTeran et al. 2003). Esse mesmo comportamento reprodutivo foi observado durante a captura de $P$. unifilis nos rios Guaporé e São Miguel. Armadilhas colocadas nas saídas das baías, nos canos e nos paranás, capturaram machos e fêmeas adultas deslocando-se para os locais de reprodução. Uma parte da população não realiza essa migração, ficando nos lagos durante a vazante. Esses deslocamentos associados ao comportamento reprodutivo influenciam a estrutura da população e a razão sexual nesses hábitats. Não temos informações sobre as populações que ficam isoladas nos lagos dentro da floresta.

O baixo número de fêmeas nos rios Guaporé e São Miguel pode refletir a captura de animais adultos durante a época de reprodução. Moradores da comunidade de Porto Mortinho, no rio São Miguel, nos informaram que as pessoas capturavam com linha cerca de 40 animais por dia durante a época seca. Segundo Soares (2000), a captura tradicional é feita pela coleta de fêmeas adultas nas praias e pela pesca de indivíduos juvenis e adultos com anzol e isca. Os pescadores profissionais locais relataram que capturaram quelônios casualmente quando passaram redes para capturar peixes.

Atividades recreativas, como camping, pesca esportiva e uso das praias por banhistas, contribuem para o aumento da predação de ninhos. Em agosto, observamos pessoas procurando covas nas praias. Esse mesmo tipo de atividade destrutiva foi relatado por FachínTeran et al. (2003) na Reserva de Desenvolvimento Sustentável Mamirauá. 

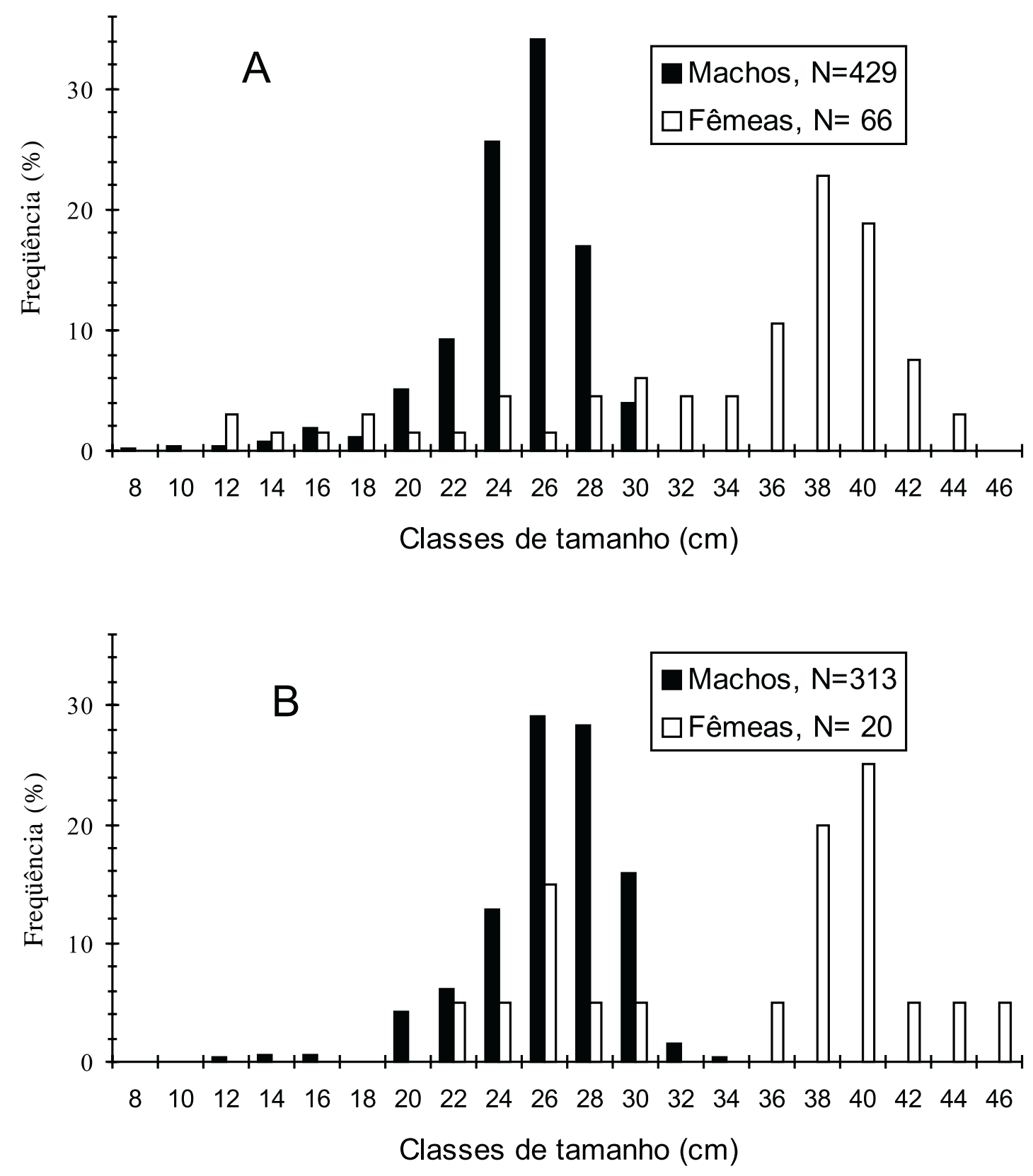

Figura 7 - Distribuição de freqüências (\%) de classes de tamanho (comprimento da carapaça) de Podocnemis unifilis em Rondônia. (A) Rio Guaporé, (B) rio São Miguel. 
Como não existe informação sobre a estrutura populacional de Podocnemis unifilis em outras localidades da Amazônia, utilizamos, para comparação, os dados de caça coletados na Reserva Mamirauá (Fachín-Terán et al. 2000). Nesse estudo, a estrutura em classes de tamanho para os machos e fêmeas apresentou uma tendência a uma curva bimodal. Entretanto, os tamanhos (comprimento linear da carapaça) mais freqüentes dos indivíduos encontrados no rio Guaporé e na Reserva Mamirauá foram similares para ambos os sexos.

Na segunda quinzena de junho, observamos pelo menos 11 filhotes de $P$. unifilis sobre galhos numa poça pouco profunda na estrada para Costa Marques. Perto desse local, corria um riacho de floresta de cerca de $20 \mathrm{~m}$ de largura. Apesar das armadilhas capturarem indivíduos acima de $5 \mathrm{~cm}$ de largura, acreditamos que a captura de poucos indivíduos de tamanho pequeno pode ter sido devida a preferências de micro-hábitats nessa fase da vida. Outro fator que poderia ter influenciado é a alta taxa de perda de ninhos por predação antrópica, além da mortalidade nas primeiras fases da vida por predação por diversos vertebrados, como as aves Phaetusa simplex (Laridae), Egretta alba, Ardeia cocoi (Ardeidae), Mycteria americana, Jaburu mycteria (Ciconidae), o jacaré Caiman crocodilus (Alligatoridae) e os peixes do gênero Serrasalmus (Serrasalmidae), abundantes no rio Guaporé e tributários.

Diferentes métodos de captura vêm sendo usados nos estudos das diferentes espécies de quelônios amazônicos. Bataus (1998) utilizou anzóis para capturar $P$. expansa no rio Crixásaçu (GO). Esse método não provoca a morte dos indivíduos, mas causa danos na boca dos animais. Fachín-Terán et al. (2003) usaram redes malhadeiras artesanais e redes transmalhas (trammel net) durante seu estudo com $P$. sextuberculata na Reserva Mamirauá. A taxa de mortalidade foi relativamente baixa $(2,52 \%)$, o que revelou que as malhadeiras deveriam ser revisadas em intervalos regulares de quatro horas. Neste estudo, foram usadas armadilhas do tipo fyke net revisadas a cada 12 horas, e, apesar da taxa de mortalidade ter sido ser relativamente baixa $(1,1 \%)$, visitas mais freqüentes às armadilhas poderiam reduzir ainda mais a mortalidade dos animais.

Mudanças na razão sexual das populações de Podocnemis podem ser o resultado de diferentes fatores ou uma combinação deles, incluindo (1) a predação diferencial histórica de fêmeas nas praias de desova (Ramo 1982, Bataus 1998, Fachín-Terán et al. 2003), (2) a influência de fatores ambientais, como baixas e/ou altas temperaturas durante o período crítico de determinação do sexo, diferenças na temperatura de incubação entre as estações, praias baixas com alta umidade e baixas temperaturas, textura da areia, profundidade do ninho (Souza e Vogt 1994, Pezzuti e Vogt 1999), (3) a captura artesanal e comercial nos locais de alimentação e de reprodução e (4) a idade na maturidade sexual (Fachín-Terán et al. 2003).

Usando dados de caça coletados no município de Costa Marques (RO), Soares (2000) relatou uma razão sexual de 1:14 a favor das fêmeas para $P$. expansa e de 1:9 para $P$. unifilis. Esse desvio a favor das fêmeas em $P$. expansa foi confirmado por este autor por meio de capturas no rio Guaporé e pelo exame do sexo dos filhotes, sendo a razão sexual de 1:22 e $1: 12$, respectivamente. Os dados de Soares (2000) para $P$. expansa são contrastantes com os encontrados para $P$. unifilis neste estudo para essa mesma região, onde a razão sexual baseada em todos os indivíduos que alcançaram a maturidade sexual foi de 9,8:1 a favor dos machos. Deve-se ressaltar o fato de que ambas as espécies têm determinação do sexo dependente da temperatura de incubação dos ovos; então, a razão sexual deveria ser desviada para o mesmo sexo em ambas as espécies. Provavelmente isso não acontece pelo fato de que os ninhos de $P$. expansa são construídos em praias altas com substratos que absorvem e mantêm altas temperaturas, o que possibilita uma maior produção de fêmeas. No caso de $P$. unifilis, os 
poucos ninhos que escapam à predação do homem e à destruição pelas fêmeas de $P$. expansa são deixados perto da vegetação, em locais sombreados, ou na beira de lagos e canos, onde o substrato é de folhas secas ou terra argilosa, as temperaturas são baixas e a exposição do ninho à luz é menor, produzindo, assim, mais machos que fêmeas.

No rio Trombetas (PA), a razão sexual de Podocnemis unifilis foi de 4:1 a favor das fêmeas (Fachín-Terán et al. 2003). Esses autores relataram, para $P$. sextuberculata na Reserva Mamirauá, uma razão sexual baseada em todos os indivíduos da população de 1,87:1 a favor dos machos, atribuindo esse desvio à captura das fêmeas pelo homem quando estas saem para desovar. No rio Guaporé e tributários, fatores ambientais, como baixas temperaturas durante o período critico de determinação do sexo ou predação diferencial de fêmeas em nidificação pelo homem, podem ser ter sido a causa da razão sexual de 8,4:1 desviada para os machos.

Bataus (1998) capturou, no rio Crixás-Açu (GO), animais sexualmente maduros de $P$. expansa na véspera da nidificação, relatando uma razão sexual de 3:1 a favor dos machos. No rio Guaporé, uma população dessa espécie apresentou uma razão sexual de 1:22 a favor das fêmeas (Soares 2000). Desconhecemos os fatores que poderiam explicar essas diferenças contrastantes. Bury (1979), ao analisar 39 estudos cujas amostras eram maiores que 100 indivíduos e cuja razão sexual foi de 1:1, sugeriu cautela para se aceitar taxas desiguais na proporção de machos e fêmeas. Após analisar trabalhos que relatavam razões sexuais desviadas para as fêmeas em diferentes espécies de quelônios, Gibbons (1970) fez ressalvas sobre as metodologias adotadas, considerando-as impróprias, e sobre as técnicas de amostragem, consideradas seletivas. Neste estudo, as armadilhas fyke net usadas foram seletivas para animais pequenos cujos tamanhos estavam acima de $5 \mathrm{~cm}$ de largura da carapaça. Dada a baixa taxa de mortalidade registrada $(1,1 \%)$, acreditamos que a metodologia usada seja a mais adequada para o estudo dessa espécie. No entanto, não descartamos o uso de redes malhadeiras artesanais e transmalhas como alternativas, mas sugerimos um cuidadoso uso dessas técnicas de captura e uma diminuição do tempo para sua revisão.

\section{Agradecimentos}

Aos nossos assistentes de campo e barqueiros Renato dos Santos, W. Mendes, M. Torres, C. Ticona, J. de Souza, F.E.S. Machado e Martha Gómez, por sua ajuda durante a coleta de dados. Gentil Cessel proporcionou hospedagem, alimentação e facilidades para o trabalho em sua fazenda Nazaré. Fachín-Terán recebeu uma bolsa de mestrado do $\mathrm{CNPq}$ durante sua permanência no Curso de Ecologia, INPA/UFAM. O IBAMA e a Secretaria de Agricultura de Costa Marques providenciaram suporte logístico durante a realização deste estudo. Maria de Fátima Soares Gómez deu-nos a oportunidade para a pesquisa no rio Guaporé, proporcionando suporte em todas as fases do trabalho de campo. A Miriam E. Lima de Fachín e Gabriel W. Fachín Lima, pela ajuda, estímulo, apoio em todos os instantes do trabalho de campo e pela ajuda com suas sugestões e com o Português.

\section{Referências Bibliográficas}

Alho, C. J. R., T. M. S. Danni e L. F. M. Padua. 1984. Influência da temperatura de incubação na determinação do sexo da tartaruga da Amazônia Podocnemis expansa (Testudinata, Pelomedusidae). Revista Brasileira de Biologia 44: 305-311.

Alho, C. J. R., T. M. S. Danni and L. F. M. Padua. 1985. Temperature-dependent sex determination in Podocnemis expansa (Testudinata: Pelomedusidae). Biotropica 17: 75-78.

Analytical Software. 2000. Statistix for Windows. Analytical Software. 333 pp.

Bataus, Y. S. L. 1998. Estimativa de parâmetros populacionais de Podocnemis expansa (Tartaruga-da-Amazônia) no rio Crixás-açu (GO) a partir de dados biométricos. 
Dissertação de Mestrado Não-publicada. Universidade Federal de Goiás, Brasil.

Bury, R. B. 1979. Population ecology of freshwater turtles. Pp. 571-602 in M. Harless and H. Morlock (eds.), Turtles - perspective and research. New York. John Wiley and Sons.

Cagle, F. R. 1939. A system of marking turtles for future identification. Copeia 1939: 170-173.

Cantarelli, V. H. 1997. The amazon turtles: conservation and management in Brazil. Pp. 407-410 in Proceedings: Conservation, Restoration and Management of Tortoises and Turtles. An International Conference. New York and Tortoise Society.

Fachín-Terán, A. 2003. Preservação de quelônios aquáticos com participação comunitária na Reserva de Desenvolvimento Sustentável Mamirauá, Amazonas, Brasil. Pp. 145-176 in C. Campos-Rozo e A. Ulloa (eds.), Fauna Socializada - tendencias en el manejo participativo de la fauna en América Latina. Fundación Natura, MacArthur Foundation, Instituto Colombiano de Antropología e Historia. Bogota.

Fachín-Terán, A e E. M Von Mülhen. 2003. Reproducción de la taricaya Podocnemis unifilis Troschel 1848 (Testudines: Podocnemididae) en la várzea del medio Solimões, Amazonas, Brasil. Ecología Aplicada 2: 125-132.

Fachín-Terán, A., R. C. Vogt e J. B. Thorbjarnarson. 2000. Padrões de caça e uso de quelônios na Reserva de Desenvolvimento Sustentável Mamirauá, Amazonas, Brasil. Pp. 323-337 in E. Cabrera, C. Mercolli e R. Resquin (eds.), Manejo de Fauna Silvestre en Amazonía y Latinoamérica. Asunción, Paraguay.

Fachín-Terán, A., R. C. Vogt e J. B. Thorbjarnarson. 2003. Estrutura populacional, proporção de sexos e abundância de Podocnemis sextuberculata (Testudines, Podocnemididae) na Reserva de Desenvolvimento Sustentável Mamirauá, Amazonas, Brasil. Phyllomedusa 2: 43-63.

Gibbons, J. W. 1970. Sex ratio in turtles. Research in Population Ecology 12: 252-254.

Gibbons, J. W. 1990. Sex ratios and their significance among turtle populations. Pp.171-182 in J. W. Gibbons (ed.), Life History and Ecology of the Slider Turtle. Washington, D.C. and London. Smithsonian Institution Press.

Medem, F. 1976. Recomendaciones respecto a contar el escamado y tomar las dimensiones de nidos, huevos y ejemplares de los Crocodylia y Testudines. Lozania 20 : $1-17$.
Páez, V. P. e B. C. Bock. 1998. Temperature effect on incubation period in the Yellow-Spotted River Turtle Podocnemis unifilis, in the Colombian Amazon. Chelonian Conservation and Biology 3: 31-36.

Pezzuti, J. C. B e R. C. Vogt. 1999. Nesting ecology of Podocnemis sextuberculata (Testudines, Pelomedusidae) in the Japurá River, Amazonas, Brasil. Chelonian Conservation and Biology 3: 419-424.

Ponce, M. 1979. Podocnemis unifilis Troschel 1848 "taricaya" (Chelonia, Pleurodira, Pelomedusidae) en el Bosque Nacional "Alexander von Humboldt", LoretoPerú. Monografia Não-publicada. Universidad Nacional Agraria. La Molina, Lima, Peru.

Pritchard, P.C.H. e P. Trebbau. 1984. The Turtles of Venezuela. Oxford, Ohio. Society for the Study of Amphibians and Reptiles. 414 pp.

Ramo, C. 1982. Biología del Galápago (Podocnemis vogli Müller, 1935) en el Hato El Frío, Llanos de Apure, Venezuela. Doñana, Acta Vertebrata 9: 1-161.

Rebelo, G. H. e L. Lugli. 1996. The conservation of freshwater turtles and the dwellers of the Amazonian Jaú National Park (Brazil). Pp.253-258 in S. K. Jain (ed.), Ethnobiology in Human Welfare. New Delhi, Deep Publications.

Rocque, C. 1968. Grande Enciclopédia da Amazônia. Amêl-Amônia Editora Ltda. Volumes III, IV, VI.

Soares, M. F. G. S. 2000. Distribuição, mortalidade e caça de Podocnemis (Testudinata, Pelomedusidae) no rio Guaporé, Rondônia, Brasil. Dissertação de Mestrado Não-publicada. Instituto de Pesquisas da Amazônia/ Fundação Universidade do Amazonas, Brasil.

Souza, R. R. e R. C. Vogt. 1994. Incubation temperature influences sex and hatchling size in the neotropical turtle Podocnemis unifilis. Journal of Herpetology 28: 453-464.

Valenzuela, N. 2001. Constant, shift and natural temperature effects on sex determination in Podocnemis expansa turtles. Ecology 82: 3010-3024.

Valenzuela, N., R. Botero e E. Martinez. 1997. Field study of sex determination in Podocnemis expansa from Colombian Amazônia. Herpetologica 53: 390-398.

Vogt, R. C. 1980. New methods for trapping aquatic turtles. Copeia 1980: 368-371.

Von Hildebrand, P., N. Bermudez e M. C. Peñuela. 1997. La Tortuga Charapa (Podocnemis expansa) en el Rio Caquetá, Amazonas, Colombia - aspectos de la biología reproductiva y técnicas para su manejo. Disloque Editores, Santafé de Bogotá, Colombia. 152 pp. 